\title{
Hierarchical Route Optimization Scheme Using Advanced Binding Update List (BUL+) for Nested Mobile Networks
}

\author{
Shayma Senan and Aisha Hassan A. Hashim \\ Faculty of Engineering, International Islamic University Malaysia, $53100 \mathrm{KL}$, \\ Malaysia \\ shay_sinan@yahoo.co.uk,aisha@iium.edu.my
}

\begin{abstract}
Supporting networks that roam as one unit is needed to provide the transparency of Internet in mobile frameworks, like cars, trains, planes, buses, etc. To accomplish this, NEMO (Network Mobility) Basic Support protocol has been proposed and developed by Internet Engineering Task Force (IETF). Although, it achieves continuous, optimal and secure communication to and from all nodes, it still suffers from many drawbacks, especially when the level of nesting increases. To overcome these limitations, this paper presents a new route optimization scheme for nested mobile network using hierarchical structure with Advanced Binding Update List (BUL+). From performance evaluation, it shows that this scheme reduces packet overhead, handoff latency, packet transmission delay, and achieves optimal routing.
\end{abstract}

Keywords: Mobile IPv6, Network Mobility (NEMO), Nested mobile networks, Route Optimization

\section{Introduction}

With the development of Wireless technology industry, more and more devices are needed to remain connected to the network even when they are roaming. As accessing the Internet expects to be more ubiquitous, requests for mobility are not limited to individual units nowadays. Supporting mobility of networks, that move as one set, is needed to allow the transparent provision of Internet access in mobile platforms, such as buses, trains, planes, etc. [1]

One implementation of network mobility concept is NEMO (Network Mobility) Basic Support Protocol [2] developed by the IETF (Internet Engineering Task Force). NEMO BS operates in IP layer and it's based upon Mobile IPv6 [3]. One or more mobile routers (MRs) can be included in a mobile network in order to enable access to the Internet [4]. The current point of attachment of the entire mobile network is hidden by MR [5].

Unlike MIPv6, a NEMO MR could attach to the Internet via another MR. When MRs inter-connect in this way they form a network topology known as a Nested Mobile Network. The hierarchy of mobile routers in nested NEMO complicates the selection of the route and/or router for Mobile Network nodes (MNN). New tunnel between the MR and its Home Agent (HA) will be involved for each level of a Mobile Network [6].

NEMO BS has some fundamental problems such as non-optimal routing path and high delay due to packet forwarding by $\mathrm{HA}$ and header overhead due to tunneling. Furthermore, the problem expands with each level of the nested mobile networks. As a matter of fact, transmitted data must visit the HAs of all MRs in the way to their destination (pinball routing problem, Figure 1). In addition, high handoff latency may be caused by IP-in-IP encapsulations leading to packet losses and disconnections [7]. 


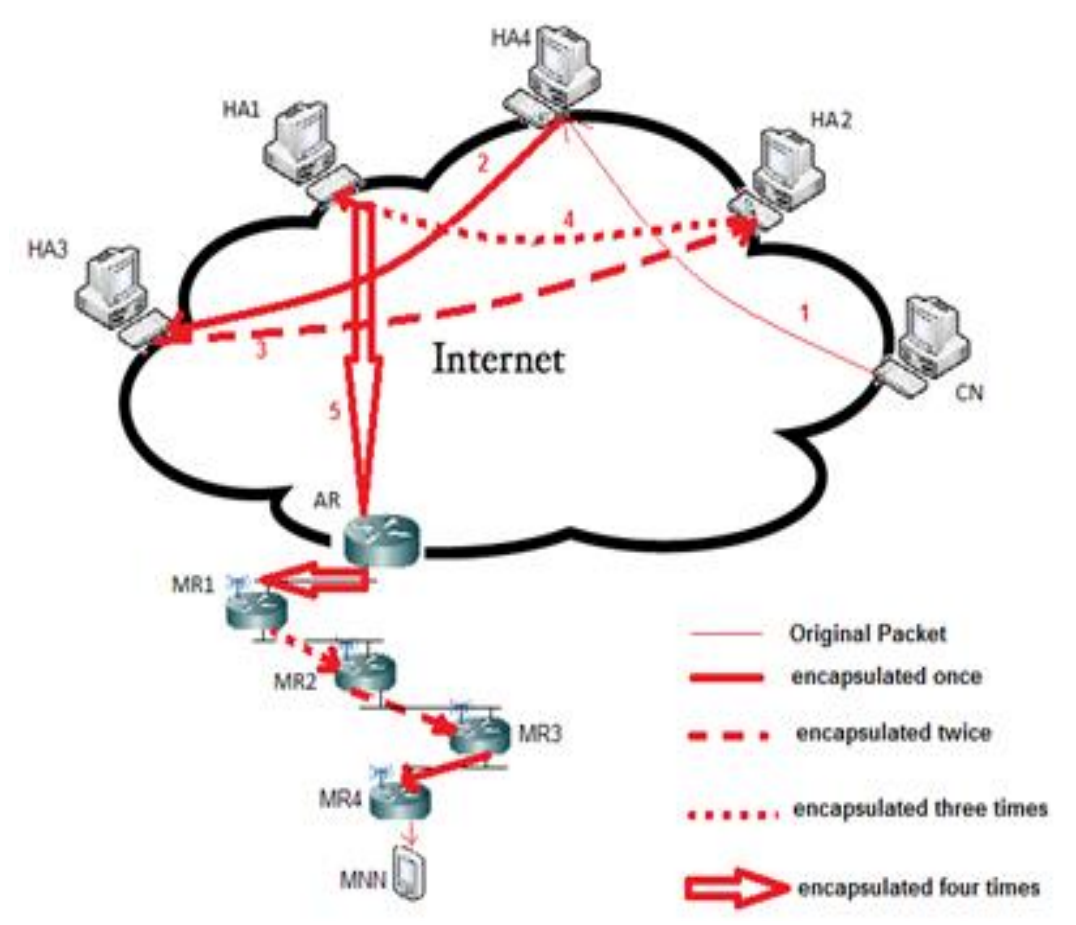

Figure 1. Non-optimal Routing Problem in Nested Mobile Networks

In this paper, a new route optimization scheme is introduced which is based on hierarchical structure using Advanced Binding Update List (BUL+) to provide an efficient NEMO with optimal routing and seamless handoff. The reminder of this paper is organized as follows. In Section 2, related works to this research are discussed. Section 3 covers the design of the proposed scheme. In Section 4, performance evaluation of NEMO BS, ROTIO, and the proposed scheme, is displayed and analyzed. The obtained results are discussed in Section 5. Finally, Section 6 concludes the research findings.

\section{Related Works}

Many studies and efforts have been done to solve the problems encountered by Mobile Networks and to present a more secure and efficient NEMO protocol. A hierarchical careof prefix (CoP) with the binding update tree (BUT) scheme, which is called HCoP-B is proposed [7] to resolve pinball routing and route optimization storm problems for the nested mobile networks. In HCoP-B, MNNs in the nested mobile networks provide shorter buffering time and shorter disruption time for ongoing real-time applications. As a result, packets that are redirected to MNN at its new location after handoff will be stored in smaller buffer spaces. But this scheme suffers from long handoff latency and packet loss during handoff. To improve the scheme and solve these problems, they proposed the predictive fast HCoP-B (FHCoP-B) [8] and then the Reactive FHCoP-B [9]. Both achieved short handoff latency and reduced packet loss, but still cannot solve the big caching size of TLMR which is considered as bottleneck to the whole mobile network.

Based on NEMO BS, Route Optimization using Tree Information Option (ROTIO) [4] proposed a routing optimization scheme with the extended tree information option (xTIO). ROTIO provides mobile networks with mobility transparency and location privacy. In addition, this scheme enables seamless handoff support and intra-NEMO route optimization. ROTIO has several disadvantages such as the non-optimal routing, MRs binding cache sizes and increased packet overhead.

Another solution to pinball routing problem and multiple levels encapsulations is a routing optimization scheme based on hierarchical MIPv6 called HRO [10]. In this 
scheme, the MAP is responsible for managing location handover, and thereby MAP domain deals with the most signaling messages locally.

Route optimization scheme in [11] provides two types of nodes: CRs (Corresponding Routers) and OLFNs (Optimization-capable Local Fixed Nodes). Since CR node is unavailable in the network always, this scheme may not be able to handle route optimization.

In [12], they proposed a new route optimization scheme by using two CoAs for each MR, as well as two types of entries in every MR's routing table. Regardless the nesting degree in the nested mobile networks, this optimized routing solution eliminates the tunnels altogether using only one BU message. Although the scheme achieves an optimal route and solve binding update storm, it's expected to have many problems during handoff especially TLMR's handoff.

Proposed schemes for NEMO BS provides solution for multi-homing, route optimization, packet overhead, handoff latency, and some of security issues. Nevertheless, nowadays there is no standardized solution that presents a coherent, complete, and integrated platform that covers all the issues of mobile networks.

\section{The Design of the Proposed Route Optimization Scheme}

The main modifications that the proposed scheme (shown in Figure 2) does to the structure of NEMO BS is in Top Level Mobile Router (TLMR) and MRs of the mobile network. No modifications are required to any of Correspondent Nodes (CNs) or Home Agents (HAs).

To localize signaling messages for handoff and optimize routing of the nested mobile networks, a Mobility Anchor Point (MAP) used in a hierarchical approach, like HMIPv6 [13], is proposed. However, for the nested mobile networks, the TLMR is proposed to be functioning like a MAP in HMIPv6. Thus, the entire nested NEMO becomes a local MAP domain. The MAP records the binding information for all MRs and MNNs, and provides optimized route from the $\mathrm{CN}$ to the $\mathrm{MNN}$ in the nested mobile networks.

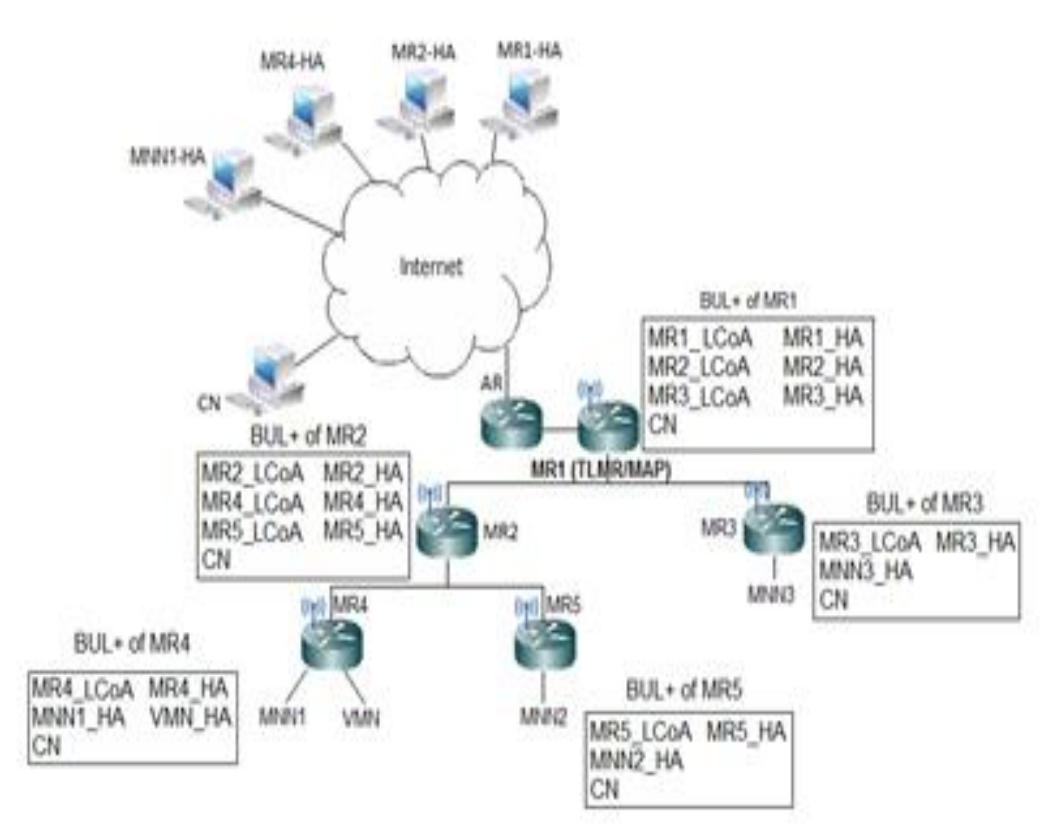

Figure 2. The Architecture of the Proposed Scheme showing BUL+ in MRs

MR in the proposed scheme is assumed to have a binding cache that can encapsulate packets and send to any node, beside sending to its HA. However, in the proposed 
scheme, a novel Advanced Binding Update List (BUL+) is introduced and built in each MRs of the nested NEMO in order to record information about all child MRs/MNNs located under each MRs. This information includes bindings sent to CNs addresses, HAs of MRs, and HAs of MNNs (more details about the operations of the proposed scheme are in [14]). The following pseudo codes shows how BUL+ is built in each MR of the nested mobile networks:

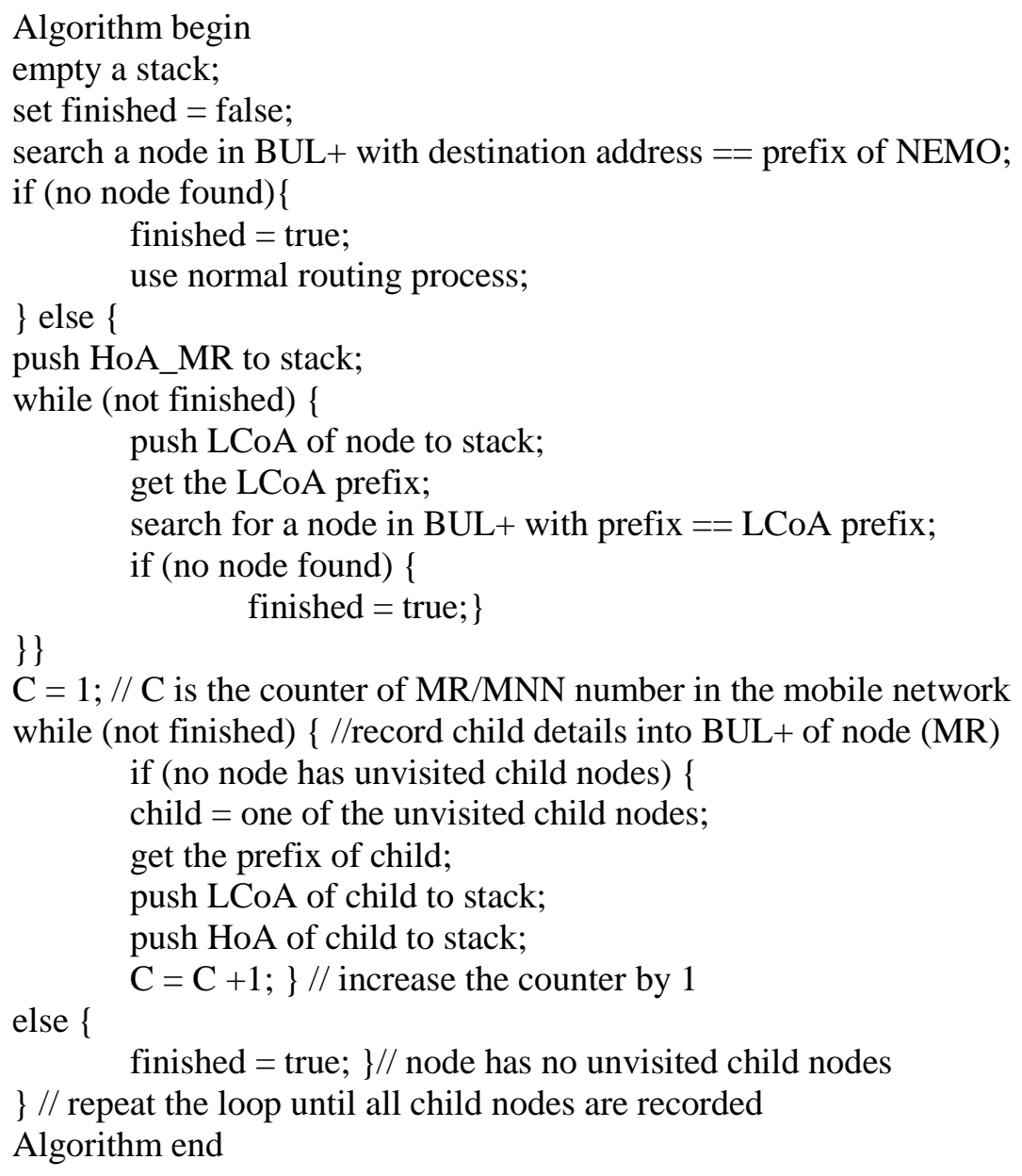

\section{Performance Evaluations}

An analytical evaluation is elaborated for the proposed scheme to test its performance and compare it with NEMO BS (the standard) and ROTIO (benchmark) [5]. The performance metrics used for this evaluation are: packet transmission delay, handoff latency and routing cost.

However, to derive the required equations for the analytical model, these notations will be used:

$\mathrm{N}$ : degrees of nesting

$\mathrm{M}$ : degrees of nesting for $\mathrm{CN}$ in Intra-NEMO

$D_{M R}^{i}$ : the $\mathrm{MR}_{\mathrm{i}}$ processing delay

$D_{H A}^{i}$ : the HA $i$ processing delay

$L D_{M R}^{i, i+1}$ : the ${ }_{M R i}-M R_{i+1}$ link delay

$L D_{\text {Router }}^{i, i+1}$ : the Router $\mathrm{i}_{\mathrm{i}}-$ Router $_{\mathrm{i}+1}$ link delay

LDHA-Router : the HA - Router link delay

LDCN-Router : the CN - Router link delay 
LDAR-TLMR : the AR - TLMR link delay

LDAR-Router : the AR - Router link delay

LDMR-MNN : the MR - MNN link delay

DMD : delay of mobility detection

DDAD : delay of DAD (duplicate address detection)

\subsection{Packet Transmission Delay}

NEMO BS does not take route optimization into account as all packets must go through the bidirectional tunnelling between MR and its HA.

The total packet delay from $\mathrm{CN}$ to $\mathrm{MNN}$ in NEMO can be calculated using the following equation:

$$
\begin{gathered}
\mathrm{PD}_{\mathrm{NEMO}}=\left(\mathrm{LD}_{\mathrm{CN}-\mathrm{Router}}+\mathrm{LD}_{\mathrm{HA}-\mathrm{Router}}\right)+2 \sum_{i=1}^{N} \mathrm{LD}_{\mathrm{HA}-\mathrm{Router}}+\sum_{i=1}^{N} \mathrm{LD}_{\text {Router }}^{i, i+1}+\mathrm{LD}_{\mathrm{AR}-\mathrm{Router}}+ \\
\mathrm{LD}_{\mathrm{AR}-\mathrm{TLMR}}+\sum_{i=1}^{N}\left(D_{H A+}^{i} D_{M R}^{i}+L D_{M R}^{i, i+1}\right)+\mathrm{LD}_{\mathrm{MR}-\mathrm{MNN}}
\end{gathered}
$$

Unlike NEMO, ROTIO supports route optimization with 2-level of encapsulation. The total packet delay in ROTIO can be calculated as the following:

$$
\begin{gathered}
\mathrm{PD}_{\mathrm{ROTIO}}=\left(\mathrm{LD}_{\mathrm{CN}-\mathrm{Router}}+\mathrm{LD}_{\mathrm{HA}-\text { Router }}\right)+2 \sum_{i=1}^{a-1} \mathrm{LD}_{\text {HA-Router }}+\sum_{i=1}^{a-1} \mathrm{LD}_{\text {Router }}^{i, i+1}+\sum_{i=1}^{a}\left(D_{H A}^{i}\right)+ \\
\mathrm{LD}_{\mathrm{AR}-\mathrm{Router}}+\mathrm{LD}_{\mathrm{AR}-\mathrm{TLMR}}+\sum_{i=1}^{N}\left(D_{M R}^{i}+L D_{M R}^{i, i+1}\right)+\mathrm{LD}_{\mathrm{MR}-\mathrm{MNN}}
\end{gathered}
$$

Where $\mathrm{a}=2$ always in ROTIO.

For the proposed scheme, it supports route optimization but with 1-level of encapsulation. The total packet delay in the proposed scheme is measured by:

$$
\begin{gathered}
\mathrm{PD}_{\text {proposed }}=\left(\mathrm{LD}_{\mathrm{CN}-\mathrm{Router}}+\mathrm{LD}_{\mathrm{HA}-\text { Router }}\right)+\sum_{i=1}^{a}\left(D_{H A)+}^{i}+\mathrm{LD}_{\mathrm{AR}-\mathrm{Router}}+\mathrm{LD}_{\mathrm{AR}-\mathrm{TLMR}}+\sum_{i=1}^{N}\left(D_{M R}^{i}\right.\right. \\
\left.+L D_{M R}^{i, i+1}\right)+\mathrm{LD}_{\mathrm{MR}-\mathrm{MNN}}
\end{gathered}
$$

Where $\mathrm{a}=1$ always in the proposed scheme.

Resulting from equations (1), (2) and (3) and by applying numerical values shown in Table 1, Figure 3 presents the packet transmission delay of the proposed scheme compared to that in NEMO BS and ROTIO. it can be seen that packet delay for NEMO increased dramatically by the increase of the nesting level and it's much higher than the delay of both ROTIO and the proposed scheme because the packets in NEMO have to go through the long path from $\mathrm{CN}$ to $\mathrm{MNN}$ and vice versa, while the proposed scheme adopts route optimization that reduces the packet delay. It is clear also that the proposed scheme has less delay than ROTIO, since ROTIO uses route optimization with 2-level of encapsulation unlike the proposed scheme that uses 1-level only.

Table 1. Parameter Values for Numerical Analysis

\begin{tabular}{|c|c|}
\hline Parameter & Value \\
\hline$D_{M R}^{i}$ & $10 \mathrm{~ms}$ \\
\hline$L D_{M R}^{i, i+1}$ & $5 \mathrm{~ms}$ \\
\hline$L D_{\text {Router }}^{i, i+1}$ & $5 \mathrm{~ms}$ \\
\hline$D_{\text {HA }}^{i}$ & $10 \mathrm{~ms}$ \\
\hline LD & $50 \mathrm{~ms}$ \\
\hline LD $_{\text {HA-Router }}$ & $10 \mathrm{~ms}$ \\
\hline LD $_{\text {MR-MNN }}$ & $5 \mathrm{~ms}$ \\
\hline LD $_{\text {AR-Router }}$ & $5 \mathrm{~ms}$ \\
\hline LD $_{\text {AR-TLMR }}$ & $100 \mathrm{~ms}$ \\
\hline$D_{\text {MD }}$ & $50 \mathrm{~ms}$ \\
\hline $\mathrm{D}_{\text {DAD }}$ & $100 \mathrm{~ms}$ \\
\hline
\end{tabular}




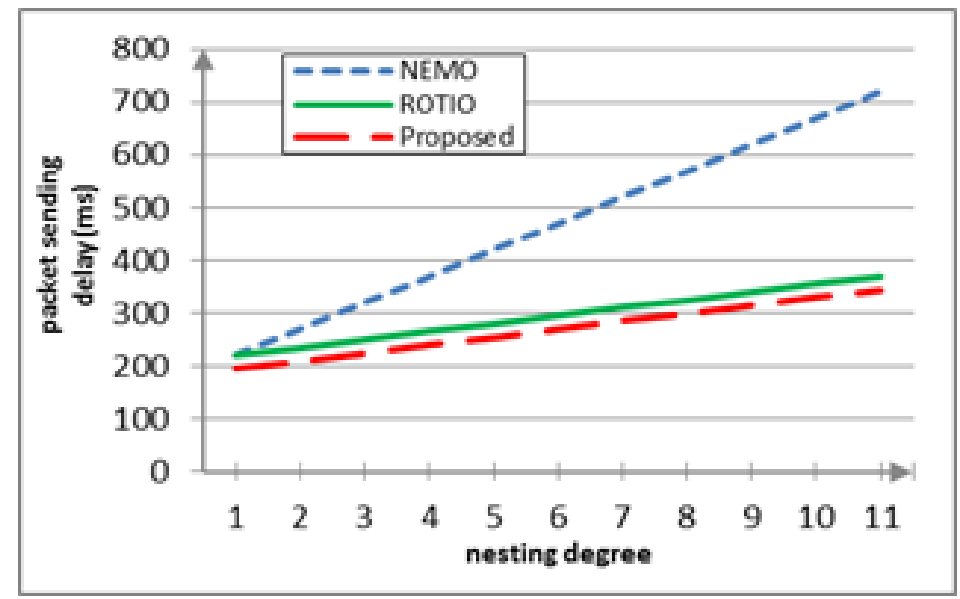

Figure 3. Packet Transmission Delay with different Levels of Nesting

\subsection{Handoff Latency}

For NEMO, the MR performs the intra-NEMO handoff procedure when it changes its point of attachment in the same nested NEMO. In NEMO BS protocol, the intra-NEMO handoff latency is the longest as it doesn't support this type of handoff. Thus it relies on MIPv6 in case of handoff.

The intra-NEMO handoff latency (HL) of NEMO is:

$$
\mathrm{HL}_{\mathrm{NEMO}}=\mathrm{D}_{\mathrm{MD}}+\mathrm{D}_{\mathrm{DAD}}+\mathrm{LD}_{\mathrm{AR}-\mathrm{HA}}+\mathrm{LD}_{\mathrm{AR}-\mathrm{MAP}}+\sum_{i=1}^{n}\left(D_{M R}^{i}\right)+\sum_{i=1}^{n-1}\left(L D_{M R}^{i, i+1}\right)
$$

The intra-NEMO handoff latency for ROTIO is:

$$
\mathrm{HL}_{\mathrm{ROTIO}}=\mathrm{D}_{\mathrm{MD}}+\mathrm{D}_{\mathrm{DAD}}+\sum_{i=1}^{n}\left(D_{M R}^{i}\right)+\sum_{i=1}^{n-1}\left(L D_{M R}^{i, i+1}\right)
$$

The proposed scheme is designed to reduce the intra-NEMO handoff latency which is presented in the following equation:

$$
\mathrm{HL}_{\text {proposed }}=\mathrm{D}_{\mathrm{MD}}+\sum_{i=1}^{n}\left(D_{M R}^{i}\right)+\sum_{i=1}^{n-1}\left(L D_{M R}^{i, i+1}\right)
$$

Using the above equations (6, 7 and 8), the handoff latency of NEMO BS, ROTIO and the proposed scheme is illustrated in Figure 4. The proposed scheme shows the lowest handoff latency because the moving MR does not perform the DAD procedure in the intra-NEMO handoff, as it configures its LCoA (Local Care-of Address) locally from MAP (TLMR). Whereas the handoff latency in NEMO protocol is the longest as it doesn't support this type of handoff. Thus it relies on MIPv6 in case of handoff. 


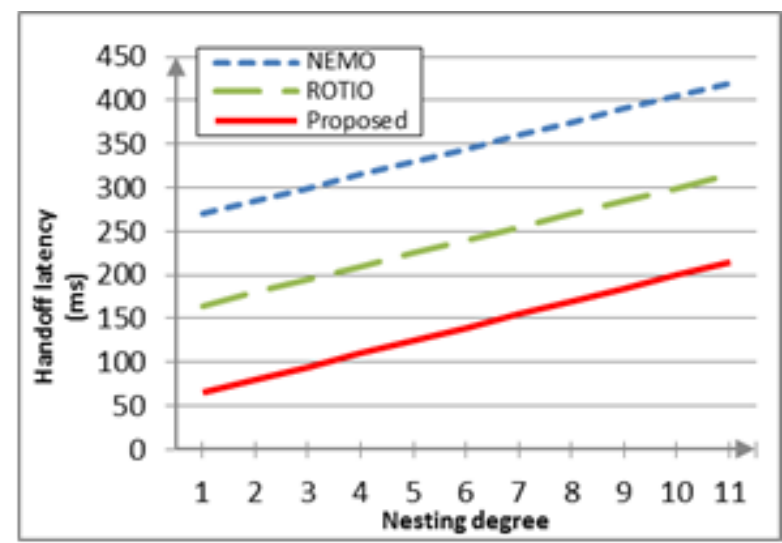

Figure 4. Handoff Latency of NEMO BS, ROTIO and the Proposed Scheme

\subsection{Routing cost}

Routing cost (C) is represented by the link delay, since HAs could be scattered all over the world causing long routing distance. To calculate the routing cost of NEMO, ROTIO, and the proposed scheme, the following notations are used:

$\mathrm{N}$ : nesting degree

$\mathrm{C}_{\mathrm{i}}$ : routing cost between the HAs of $\mathrm{MR}_{\mathrm{i}}$ and $\mathrm{MR}_{(\mathrm{i}+1)}$

$\mathrm{C}_{\mathrm{N}}$ : routing cost between the HA of TLMR and the TLMR of the mobile network

$c_{i}$ : routing cost between the $C N$ and the $H A$ of the $M_{R}\left(M R_{N}\right.$ is TLMR)

However, routing cost $\left(\mathrm{C}_{\mathrm{NEMO}}\right)$ in NEMO BS (using bi-directional tunneling) is measured by:

$$
\mathrm{C}_{\mathrm{NEMO}}=\mathrm{c}_{\mathrm{i}}+\sum_{i=1}^{N-1} \mathrm{Ci}
$$

For ROTIO, routing cost $\left(\mathrm{C}_{\mathrm{ROTIO}}\right)$ can be calculated as follows:

$$
\mathrm{C}_{\mathrm{ROTIO}}=\mathrm{c}_{\mathrm{i}}+\mathrm{C}_{1}+\mathrm{C}_{\mathrm{N}}
$$

Lastly, routing cost in the proposed scheme $\left(\mathrm{C}_{\text {Proposed }}\right)$ can be measured as follows:

$$
\mathrm{C}_{\text {Proposed }}=\mathrm{c}_{\mathrm{i}}+\mathrm{C}_{\mathrm{N}}
$$

As shown in above equations, for large number of nesting level, the routing cost for bidirectional tunneling increases linearly. However, as shown in Figure 5, for ROTIO and the proposed scheme, the routing cost remains constant because there will always be 2 level of tunneling (for ROTIO) and 1-level of tunneling (for proposed scheme) regardless the degree of nesting. 


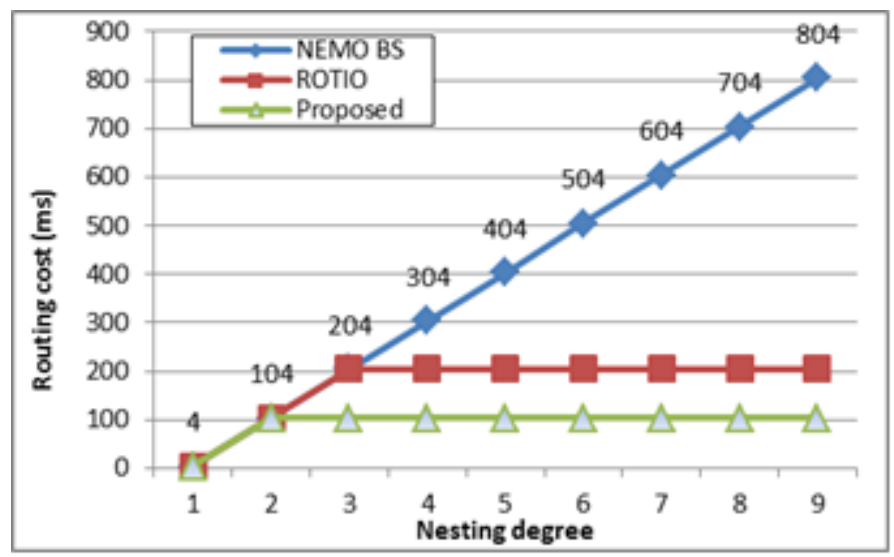

Figure 5. The Routing Cost of NEMO BS, ROTIO and the Proposed Scheme

\section{Results Discussion}

From analytical evaluation described in the previous Section, it is clear that the number of hops (HAs) involved in data communication between $\mathrm{CN}$ and MNN played a significant role on the performance of the mobile network. As a result, packet transmission delay for NEMO BS is much higher than that of ROTIO and the proposed scheme. However, the proposed scheme is $9.26 \%$ better than ROTIO and $74 \%$ better than NEMO BS.

For handoff latency, the proposed scheme has less than that in ROTIO and NEMO BS because the handoff of the MR happened within the MAP domain where the CoA is configured locally. In other words, handoff latency in the proposed scheme is $71.4 \%$ better than ROTIO and $146.4 \%$ better than NEMO BS.

Routing cost in NEMO BS increases dramatically as the level of nesting increases, while in ROTIO, it remains constant after $204 \mathrm{~ms}$ routing cost, i.e. after two level of nesting. For the proposed scheme, it remains constant because there will always be one level of tunnelling regardless the degree of nesting. However, the proposed scheme has better performance which is three times better than NEMO BS and $83.7 \%$ better than ROTIO.

However, the result proves that the proposed scheme has better performance than NEMO BS and ROTIO especially when the nesting level increases.

\section{Conclusion}

In this paper, a new route optimization scheme has been proposed and described which is based on hierarchical structure with Advanced Binding Update List (BUL+). This proposed scheme aims to solve the pinball routing problem (non-optimal routing problem) and achieve seamless handoff. This will lead to minimize transmission delay, handoff latency, packet overhead, and accomplish optimal routing. As a result, it solves the problems face NEMO Basic Support, especially in nested mobile network. The performance evaluation results show that the proposed solution has less packet transmission delay and less handoff latency compared to the standard NEMO Basic Support protocol and ROTIO protocol.

\section{References}

[1] S. Jeon and Y. Kim, "Cost-efficient Network Mobility scheme over proxy mobile IPv6 network", IET Commun., vol. 5, iss.18, (2011), pp. 2656-2661.

[2] V. Devarapalli, R. Wakikawa, A. Petrescu and P. Thubert, "NEMO basic support protocol", IETF, RFC3963, (2015), Available: http://www.ietf.org/rfc/rfc3963.txt 
[3] D. Johnson, C. Perkins and J. Arkko, "Mobility Support in IPv6", IETF, RFC6274, (2011), Available: http://www.rfc-editor.org/rfc/rfc6275.txt

[4] H. Cho, T. Kwon and Y. Choi, "Route Optimization Using Tree Information Option for Nested Mobile Networks", IEEE Journal on Selected Areas in Communications, vol. 24, no. 9, (2006), pp. 1717- 1724.

[5] F. Yousaf and C. Wietfeld, "Solving pinball routing, race condition and loop formation issues in nested mobile networks", Computer Networks, no. 56, (2012), pp. 1357-1375.

[6] M. Calderón, C. Bernardos, M. Bagnulo, I. Soto and A. de la Oliva, "Design and Experimental Evaluation of a Route Optimization Solution for NEMO", IEEE Journal on Selected Areas in Communications, vol. 24, no. 9, (2006), pp. 1702- 1716.

[7] I. C. Chang and C. H. Chou, "HCoP-B: A Hierarchical Care-of Prefix with BUT Scheme for Nested Mobile Networks", IEEE Transactions on Vehicular Technology, vol. 58, no. 6, (2009), pp. 2942-2965.

[8] I. C. Chang and C. S. Lu, "The Predictive FHCoP-B Scheme for Fast and Seamless Handoff in the Nested Mobile Network", 5th International Conference on New Trends in Information Science and Service Science (NISS), vol. 2, (2011), pp. 215 - 221.

[9] I. C. Chang, C. S. Lu and S. C. Wang, "Performance Analysis of Reactive FHCoP-B in Nested Mobile Networks", Journal of Applied Mechanics and Materials, vol. 284-287, (2013), pp. 2850-2854.

[10] $\mathrm{H}$. Wu and J. Lu, "Research on Routing Optimization in Nested NEMO", in the proceedings of the International Conference on Electronics, Communications and Control (ICECC), China, (2011), pp. 411-415.

[11] R. Kong, J. Feng, R. Gao and H. Zhou, "A new route optimization scheme for Network Mobility: combining ORC protocol with RRH and using quota mechanism", The International Journal of Communications and Networks, vol. 14, no. 1, (2012), pp. 91-103.

[12] M. H. Kabir, M. Mukhtaruzzaman and M. Atiquzzaman, "Efficient route optimization scheme for nested-NEMO", Journal of Network and Computer Applications, no. 36, (2013), pp. 1039-1049.

[13] H. Soliman, C. Castelluccia, K. ElMalki and L. Bellier, "Hierarchical Mobile IPv6 (HMIPv6) Mobility Management", IETF, RFC 5380, (2008), Available: http://www.rfc-editor.org/rfc/rfc5380.txt

[14] S. Senan, A. H. A. Hashim, A. M. Zeki, R. A. Saeed, S. A. Hameed and J. I. Daoud, "A Framework of a Route Optimization Scheme for Nested Mobile Network", Lecture Notes in Computer Science, vol. 7667, pp. 689-696. 
International Journal of Future Generation Communication and Networking Vol. 10, No. 2 (2017) 